\title{
BMJ Open Quality Analysis of patient safety messages delivered and received during clinical rounds
}

\author{
Diane Levine, ${ }^{1,2}$ Jaya Gadivemula, ${ }^{2}$ Raya Kutaimy, ${ }^{2}$ Srinivasa Kamatam, ${ }^{2}$ \\ Nagaratna Sarvadevabatla, ${ }^{2}$ Prateek Lohia ${ }^{2}$
}

To cite: Levine D, Gadivemula J, Kutaimy R, et al. Analysis of patient safety messages delivered and received during clinical rounds. BMJ Open Quality 2020;9:e000869. doi:10.1136/ bmjoq-2019-000869

Received 30 January 2020 Revised 28 May 2020 Accepted 3 July 2020
Check for updates

(C) Author(s) (or their employer(s)) 2020. Re-use permitted under CC BY-NC. No commercial re-use. See rights and permissions. Published by BMJ.

${ }^{1}$ Internal Medicine, Wayne State University, Detroit, Michigan, USA

${ }^{2}$ Internal Medicine, Detroit Medical Center, Wayne State University, Detroit, MI, USA

Correspondence to

Dr Diane Levine;

dllevine@med.wayne.edu

\section{ABSTRACT}

Background Multiple modalities are available to introduce patient safety training to healthcare professionals. In internal medicine, clinical rounds have always played an important role in education; however, the patient safety content taught at the point of care is not well studied. We studied, both quantitatively and qualitatively, the number and nature of patient safety messages delivered by attending physicians to determine what is taught at the point of care and how well this is recognised and recalled by attending physicians, residents and medical students. Methods This prospective mixed methods study was conducted on the medicine teaching service. Clinical rounds were audio-recorded. Immediately after rounds, attending physicians, residents and students completed a short survey card identifying the number and type of educational messages they immediately recalled teaching or hearing. Independent t-test was used to compare differences in the number of messages delivered by attendings and recalled by trainees. One-way analysis of variance was used to compare differences in messages delivered by attending physicians compared with trainees. Recordings were transcribed and analysed qualitatively for patient safety content.

Results Trainees recalled more educational messages than attendings recalled teaching in all educational domains. Safety messages comprised $17.5 \%$ of educational messages. The average number of patient safety messages recalled per session was 1.08 per attending physicians, 1.84 per resident and 2.50 per student. Residents recalled $56.4 \%$ of safety messages delivered; students recalled $76.7 \%$ of safety messages. Conclusion Patient safety is a focus of teaching during clinical rounds and provides meaningful opportunities to train students and residents to practice safe patient care.

\section{INTRODUCTION}

Preventable medical errors are estimated to cause 98000-440000 deaths and are the third leading cause of mortality in the USA after heart diseases and cancer. ${ }^{1}$ According to the WHO, even in developed countries, 1 in 10 hospitalised patients is harmed while receiving care. ${ }^{2}$ Integration of patient safety training across the continuum of medical education is essential to prevent medical errors. ${ }^{3}$ Both the WHO and the American Association of Medical Colleges have endorsed increased patient safety education during medical school. ${ }^{3-6}$ Patient safety content is increasingly being tested on certifying examinations including United States Medical Licensing Examinations and the National Board of Osteopathic Medical Examination. ${ }^{7}$

A cross-sectional study found that medical students have low knowledge of patient safety and quality improvement. ${ }^{8}$ A survey of US and Canadian medical schools revealed that only $46 \%$ had explicit patient safety curricula. ${ }^{9}$ With development of the Core Entrustable Professional Activities for Entering Residency, incorporating patient safety education into medical school curricula will be necessary to align with these graduation requirements that include a focus on the culture of safety and improvement. ${ }^{1011}$

Efforts are also in place to engage graduate physician trainees in patient safety activities to enhance the quality of care delivered in US teaching hospitals. ${ }^{12} 13$ The Clinical Learning and Environment Review programme, formulated by the Accreditation Council for Graduate Medical Education, focuses on graduate training programmes' learning environment and efforts to improve patient safety and healthcare quality through engagement of residents and fellows. ${ }^{14}$ Specific competencies for quality improvement and patient safety have been proposed for learners across the continuum. ${ }^{1617}$

Multiple modalities, including lecturebased curricula, online educational modules, for example, from the Institute for Health Improvement $^{18}$ and internally developed programmes, ${ }^{19-21}$ are available for patient safety training. In internal medicine, clinical rounds have always played a pivotal role in training. Bedside rounds have been studied as a way to teach history taking, physical exam skills, ethics, professionalism and communication skills ${ }^{2022} 23$ but have not been extensively studied as a vehicle to teach patient safety. There is a paucity of literature on 
patient safety education and training delivered during clinical rounds, and little attention has been given to the actual patient safety content delivered.

We studied the teaching conversations that take place during clinical rounds and analysed qualitatively to determine what is taught at the point of care with a focus on patient safety. We then evaluated immediate recall of safety messages by attending physicians, residents, and medical students and immediate recall relative to number of safety messages identified in the qualitative review.

\section{METHODS}

We conducted a mixed methods prospective cohort study during September 2012 on the Wayne State University academic internal medicine service at Detroit Receiving Hospital. Five teams participated in the study. Each team was composed of an attending physician, one senior resident, two interns, and one or two medical students.

Clinical rounds were audio-recorded daily for 1 week (Monday through Friday). A total of 24 rounding sessions were recorded. Consent was obtained prior to recording. Participants were told the research aim was to 'study teaching rounds'. The particular focus and area of interest in patient safety were not disclosed to avoid biassed attention to patient safety. Immediately after rounds, survey cards were distributed to each member of the team. Each card listed six domains of clinical teaching: cost effectiveness, evidence-based medicine (EBM), feedback, patient safety, professionalism and physical examination skills. Participants identified the number of educational messages delivered on rounds by educational domain. Survey cards were completed independently by each participant and were collected by a member of the research team. Recordings were downloaded daily and transcribed. The study received an expedited approval from the university institutional review board.

\section{Qualitative analysis}

The methodology for qualitative analysis of transcripts was derived from Strauss and Corbin's Basics of Qualitative Research (1990) ${ }^{24}$ All transcripts obtained were reviewed for teaching content. Educational messages were identified and categorised into one of six domains: cost effectiveness, EBM, feedback, professionalism, teaching physical examination and patient safety. To capture the nature of messages, investigators coded the content of the message in as many categories as appropriate. When an evidence-based guideline was referenced to teach a patient safety point, we categorised the message as both patient safety and EBM component.

Patient safety messages were further analysed. Before beginning the study, investigators reviewed the Agency of Healthcare Research and Quality's Safety Primers ${ }^{25}$ and used these topics to inform analysis of patient safety messages. A master list of all patient safety messages was created from the transcripts. Inductive codes were formulated to identify safety messages. Similar messages were pooled. The messages identified were grouped according to their respective codes. A total of 61 codes emerged during this process. These codes were grouped by their nature into eight themes related to patient safety: medication safety, discharge safety, preventing diagnostic errors, procedural safety, communication, inpatient safety, healthcare-associated infections and patient safety culture. Transcripts were studied one more time to check the appropriateness of the codes applied to the data. Discussion and consensus addressed disagreements in coding.

To determine the validity of our coding, attending physicians were contacted to review transcripts of their own rounding sessions and to identify patient safety messages. Three of five were available to provide reviews, and three transcripts each (nine total) were reviewed. The patient safety messages identified by attending physicians were compared with those identified by the research team. We also invited one full team (attending and residents) to review transcripts from their three rounding sessions. Safety messages observed from transcripts by the attending physician and residents were compared. The medical students were no longer at the institution and did not provide reviews.

\section{Quantitative analysis}

Using the survey cards, we compared the number of educational messages by domain recalled by attending physicians, residents and students. We also compared immediate recall of patient safety messages by attending physicians, residents and medical students, and immediate recall relative to the number of safety messages observed in the qualitative review of transcripts.

Using the number of messages identified by attending physicians on their transcripts, referred to as 'observed' safety messages, as the actual number of messages delivered/taught, we calculated a recall percentage (the number of messages recalled divided by number of messages observed) for residents and for students.

Independent t-tests were used to compare the differences in the number of educational messages recalled by the attending physicians, residents and medical students. One-way analysis of variance was used to compare differences in messages delivered by attending physicians and those recalled by residents and between attending physicians and those recalled by students.

\section{RESULTS}

Attending physicians recalled the delivery of 148 educational messages (EBM, 39; cost effectiveness, 22; feedback, 22; patient safety, 26; professionalism, 17; physical exam, 22) across six educational domains with an average of 6.08 ( $\mathrm{SD}=3.21)$ messages per rounding session. Residents and medical students (referred to as trainees) recalled receiving an average of $10.56(\mathrm{SD}=4.14)$ and 14.1 $(\mathrm{SD}=9.1)$ educational messages per rounding session, 
Table 1 Average number of educational messages recalled per rounding session by participants across six domains of clinical teaching

\begin{tabular}{llrr}
\hline Domain & Attending physicians (SD) & Residents (SD) & Medical students (SD) \\
\hline EBM & $1.61(0.92)$ & $2.54(1.49)$ & $3.28(1.96)$ \\
Patient safety & $1.04(0.41)$ & $1.84(0.86)$ & $2.50(1.94)$ \\
Physical exam & $0.91(0.66)$ & $1.16(0.83)$ & $1.31(1.03)$ \\
Feedback & $0.89(0.83)$ & $1.76(0.57)$ & $3.17(1.90)$ \\
Cost effectiveness & $0.89(0.56)$ & $1.92(1.05)$ & $2.28(1.07)$ \\
Professionalism & $0.70(0.68)$ & $1.35(0.62)$ & $1.54(2.14)$ \\
Total & $6.04(3.21)$ & $10.56(4.14)$ & $14.10(9.1)$ \\
\hline
\end{tabular}

EBM, evidence-based medicine.

respectively. These differences were not statistically significant.

Trainees recalled receiving more educational messages than attending physicians recalled teaching across all educational domains (cost effectiveness ( $\mathrm{t}=-2.389$, $\mathrm{p}=0.024)$, EBM $(\mathrm{t}=-1.550, \mathrm{p}=0.132)$, feedback $(\mathrm{t}=-2.147$, $\mathrm{p}=0.040$ ), patient safety $(\mathrm{t}=-1.617, \mathrm{p}=0.117)$, professionalism ( $\mathrm{t}=-1.136, \mathrm{p}=0.265)$ and physical exam $(\mathrm{t}=-0.726$, $\mathrm{p}=0.474)$ ). Students recalled more messages than residents in all educational domains. Differences between residents and students were only statistically significant for feedback messages $(\mathrm{p}=0.008)$; however, the number of messages in other domains was small. The average number of educational messages per session recalled by participants across all the domains is listed in table 1.

Five attending physicians recalled teaching 26 patient safety messages during the week with an average of 5.2 messages per attending physician. Patient safety messages were second in number only to EBM in the total number of messages delivered by attending physicians.

The average number of patient safety messages recalled per rounding session was 1.04 for attending physicians, 1.84 for residents and 2.50 for medical students. There was no statistically significant difference between the numbers of patient safety messages recalled between students and residents or by year of postgraduate training.

A total 364 safety messages were observed on qualitative analysis of transcripts with an average of 15.2 messages per rounding session. This was more than the total number of safety messages attending physicians recalled teaching (26) during the week and more than the average number (1.04) per rounding session.

Sixty-one codes emerged during analysis and were grouped into eight patient safety themes: discharge safety, medication safety, patient safety culture, procedural safety, healthcare-associated infections, preventing diagnostic errors and communication. Codes that represented more than $10 \%$ of the messages were considered 'major codes' and are displayed in table 2. Twenty-one major codes accounted for more than $60 \%$ of the safety messages identified. The major codes applied to the patient safety educational messages addressed key concepts articulated in the WHO patient safety curriculum for healthcare professionals. Medication safety $(32 \%)$ constituted the largest number of patient safety messages followed by discharge safety (20\%). More than half of the discharge safety messages focused on continuity of care. Educating the patient in the process to prevent the adverse events, an important component of patient care, constituted $44 \%$ of communication theme messages.

Attending physicians identified an average of 29.3 patient safety messages, whereas investigators observed an average of 41.3 patient safety messages per physician. The average number of patient safety education messages per session was greater for the research team (4.58) when compared with attending physicians' selfreview (3.26). Attending physicians were then asked to review the messages identified by research team as patient safety. There was no significant difference in the number of messages identified (36.2 vs 41.3, p=NS). Some educational messages identified and labelled as patient safety by the research team were not accepted as patient safety messages by attending physicians. Some messages identified by attending physicians were not identified by the research group. Overall, $87 \%$ agreement in messages was identified and in the relative frequency of patient safety themes.

Results from one rounding team reviewed are presented in table 3 . The attending physician observed a total of 25 safety messages; residents observed 16.5 safety messages from the same transcripts. Residents identified $66 \%$ of the safety messages observed on transcripts by attending physicians. Patient safety themes are listed table 3 . The most common safety messages from these transcripts were medication and discharge safety.

On review of their transcripts, attending physicians observed nearly threefold more patient safety messages per session (3.26 vs 1.08) than they immediately recalled on survey cards. The percentage of safety messages immediately recalled by residents was $56.4 \%$ ( 1.84 vs 3.26 ); the percentage recalled for students was $76.6 \%$ (2.50 vs 3.26).

A review of the transcripts revealed that patient safety messages taught by attending physicians were contextual and noticeable in three forms, enquiry type (eg, 'Is the patient on subcutaneous heparin?') and statement type (eg, 'Discontinue that medication'), or as a factual form 
Table 2 Qualitative analysis of transcripts from 24 rounding sessions for patient safety themes and codes applied for identification of PS educational messages

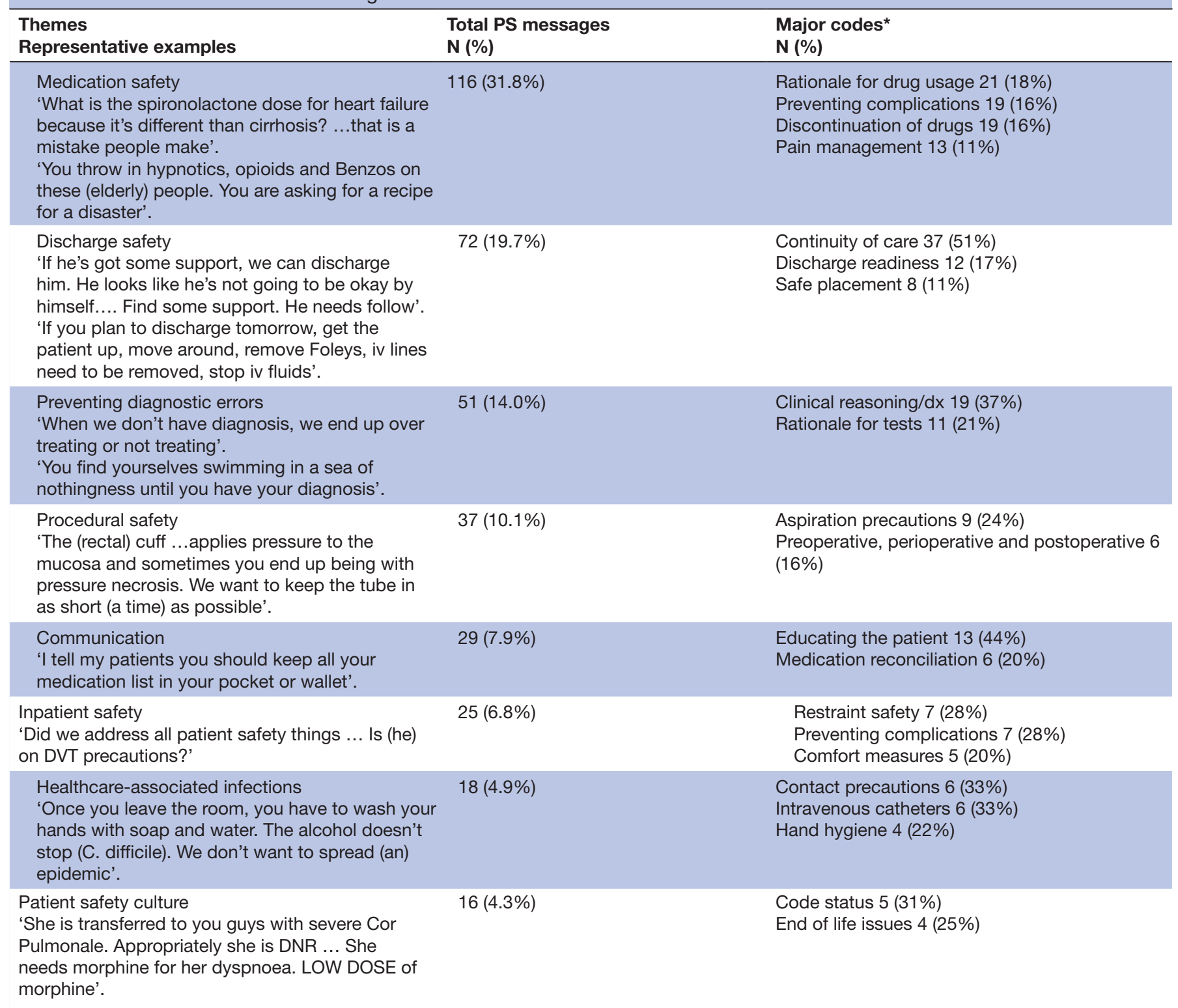

${ }^{*}$ Codes with $>10 \%$ of the frequency of messages. They accounted for $>60 \%$ of total messages.

$\mathrm{dx}$, diagnosis; PS, patient safety.

that contributed to safety either directly or indirectly (eg, 'Just make sure she is on good medication regimen before we send her out').

The number of safety messages that contained the words 'safety', 'harm' and 'careful' was less than $10 \%$ of the total. There were only two instances where the attending physician used the specific phrase 'patient safety'.

\section{DISCUSSION}

Our study showed that patient safety education is an integral part of clinical rounds. Safety education comprised $17.5 \%$ of educational messages that were delivered. Other domains of clinical education, including physical examination, cost effectiveness, professionalism and feedback, were also addressed during the rounds but less often than patient safety. Only EBM was addressed more often.

Several studies have tried to get at what is taught during clinical rounds related to patient safety. Lamba et al described real-time identification of patient safety education during rounds. ${ }^{26}$ Using direct observation, we saw a mean of eight patient safety issues per rounding team. This is more than twice what we observed. However, physicians were informed that the aim of the study was to assess how often patient safety issues occur during rounds. This may have biassed the participants, increasing the emphasis on patient safety training and the number of patient safety issues delivered. In contrast, in 'Attending rounds in the current era, what is and what is not happening', Stickrath 
Table 3 Number of PS messages by theme observed by he attending physician and residents from the same rounding team

\begin{tabular}{llc}
\hline Patient safety theme & $\begin{array}{l}\text { Attending } \\
\text { physician } \\
\text { Mean }\end{array}$ & $\begin{array}{l}\text { Residents } \\
\text { Mean }\end{array}$ \\
\hline Medication safety & $10(40 \%)$ & $4.527 \%$ \\
\hline Discharge safety & $10(40 \%)$ & $4.527 \%$ \\
\hline Diagnostic errors & 0 & $0.53 \%$ \\
\hline Procedural safety & 0 & $0.53 \%$ \\
\hline Communication & 0 & $1.59 \%$ \\
\hline Inpatient safety & $3(12 \%)$ & $318 \%$ \\
\hline Healthcare associated & $2(8 \%)$ & $212 \%$ \\
infections & 0 & 0 \\
\hline Patient safety culture & 25 & 16.5 \\
\hline Total per participant & 2.77 & 1.83 \\
\hline Average number per session & & \\
\hline
\end{tabular}

et al described the content of rounds using three domains: patient care, communication and education. ${ }^{27} \mathrm{He}$ found that patient safety issues were 'infrequently discussed and identified as a part of patient care'. In our study, we found safety was a highly represented educational domain. We did not analyse safety separate from patient care as they often occurred simultaneously as demonstrated by a detailed analysis of transcripts. We believe safety education is intimately connected with patient care. Clinical rounds provide a natural opportunity for safety education while addressing patient care issues.

We found that the patient safety education that happens during clinical rounds is integrated into routine teaching but is predominantly covert in nature. The covert nature of patient safety education happening at the point of care was evident after analysis of transcripts as the majority of patient safety messages were not identified as 'safety messages'. Instances where messages were overtly identified using specific terms like 'harm', 'prevent' and 'safety' were very uncommon.

Various types of clinical rounding practices have been studied and found to be effective in addressing core competencies. ${ }^{28}$ Bedside rounds have been studied as a method for teaching communication, physical examination, clinical reasoning and professionalism. ${ }^{20229-32}$ Our study suggests teaching patient safety can also be accomplished during bedside rounds.

Our study is unique as it provides information on the content of patient safety education generally taught at the point of care. The educational content delivered addresses all aspects of safe patient care extending from admission to postdischarge care. Analysis of rounds based on patient safety themes demonstrated that nearly all domains of patient safety training as described by the WHO in their patient safety curriculum guide topics were addressed during rounds. ${ }^{28}$
This study has several limitations. We did not prime participants to look for patient safety content, and therefore, trainees may have missed identifying patient safety messages. We also did not have participants tally messages as they were delivered, introducing the possibility of recall bias; participants may have recalled only overt messages only and have not identified covert patient safety messages delivered during the rounds. Our results represent a single institution and may not be generalisable to other training programmes. However, the teaching faculty in our study had a wide range of teaching experience and included faculty trained in both university and community settings. Attending physicians who rounded were generally aware of the importance of patient safety; none of them had specific expertise in patient safety education and likely represent many teaching attending physicians in the USA.

\section{Next steps}

We used the results of this study to educate our faculty on the opportunities clinical rounds present for integrating patient safety education into clinical care and of the importance of labelling patient safety teaching. We also found that understanding what is taught on rounds related to patient safety provides opportunities for quality improvement projects related to improve safety practices.

Our study was conducted in 2012. Many years have elapsed, but clinical rounds continue to be an important activity for trainees. We plan to restudy clinical rounds to see how the patient safety teaching at the point of care has changed over time so that we can capitalise on clinical activities that occur every day in postgraduate training.

In conclusion, this study demonstrates that patient safety has been a focus of teaching during attending clinical rounds, and patient safety teaching is recognised by residents and students. Clinical rounds provide meaningful opportunities to develop a culture of safety and train students and residents to practice safe patient care. Twitter Diane Levine @Diane Levine@DianeLevine1

Acknowledgements Fred Upton MA, Research Design and Analysis Consulting Unit of Wayne State University provided assistance in the statistical analysis of the data.

Contributors All authors had full access to the data in the study and take responsibility for the integrity of the data and the accuracy of the data analysis. Study concept and design: DL, PL and SK. Acquisition of data: JG, SK and NS. Analysis and interpretation of data: JG, SK, PL and DL. Drafting of the manuscript: JG, RK, SK and DL. Critical review of the manuscript for important intellectual content: all authors. Obtained funding: none. Study supervision: DL.

Funding The authors have not declared a specific grant for this research from any funding agency in the public, commercial or not-for-profit sectors.

Competing interests None declared.

Patient and public involvement Patients and/or the public were not involved in the design, conduct, reporting or dissemination plans of this research.

Patient consent for publication Not required.

Provenance and peer review Not commissioned; externally peer reviewed.

Data availability statement All data relevant to the study are included in the article or uploaded as supplementary information. 
Open access This is an open access article distributed in accordance with the Creative Commons Attribution Non Commercial (CC BY-NC 4.0) license, which permits others to distribute, remix, adapt, build upon this work non-commercially, and license their derivative works on different terms, provided the original work is properly cited, appropriate credit is given, any changes made indicated, and the use is non-commercial. See: http://creativecommons.org/licenses/by-nc/4.0/.

\section{REFERENCES}

1 James JT, new A. A new, evidence-based estimate of patient harms associated with hospital care. J Patient Saf 2013;9:122-8.

2 WHO. 10 facts on patient safety. Available: https://www.who.int/ features/factfiles/patient safety/en/

3 Nilsen K. Medical students' perception of patient safety-and what they learn about it: UiT Norges arktiske universitet, 2015

4 Walton M, Woodward H, Van Staalduinen S, et al. The WHO patient safety curriculum guide for medical schools. Qual Saf Health Care 2010;19:542-6.

5 Headrick L, Baron R, Pingleton S, et al. Teaching for quality: integrating quality improvement and patient safety across the continuum of medical education. Washington, DC: Association of American Medical Colleges (AAMC), 2013.

6 Kirkman MA, Sevdalis N, Arora S, et al. The outcomes of recent patient safety education interventions for trainee physicians and medical students: a systematic review. BMJ Open 2015;5:e007705.

7 Jain CC, Aiyer MK, Murphy E, et al. A national assessment on patient safety curricula in undergraduate medical education: results from the 2012 clerkship directors in internal medicine survey. J Patient Saf 2015;16:14-18.

8 Blasiak RC, Stokes CL, Meyerhoff KL, et al. A cross-sectional study of medical students' knowledge of patient safety and quality improvement. N C Med J 2014;75:15-20.

9 Alper E, Rosenberg El, O'Brien KE, et al. Patient safety education at U.S. and Canadian medical schools: results from the 2006 clerkship directors in internal medicine survey. Acad Med 2009;84:1672-6.

10 Caverzagie KJ, Cooney TG, Hemmer PA, et al. The development of entrustable professional activities for internal medicine residency training: a report from the education redesign Committee of the alliance for academic internal medicine. Acad Med 2015;90:479-84.

11 Kutaimy R, Zhang L, Blok D, et al. Integrating patient safety education into early medical education utilizing cadaver, sponges, and an inter-professional team. BMC Med Educ 2018;18:215.

12 Myers JS, Nash DB. Graduate medical education's new focus on resident engagement in quality and safety: will it transform the culture of teaching hospitals? Acad Med 2014;89:1328-30.

13 Jansma JD, Wagner C, Bijnen AB. A patient safety curriculum for medical residents based on the perspectives of residents and supervisors. J Patient Saf 2011;7:99-105.

14 Weiss KB, Bagian JP, Nasca TJ. The clinical learning environment: the foundation of graduate medical education. JAMA 2013;309:1687-8.
15 Koh NJ, Wagner R, Newton RC, et al. The CLER national report of findings 2018: trends in the CLER focus areas. J Grad Med Educ 2018;10:69-76.

16 AAMC QIPS competencies, 2019. Available: https://www.aamc.org/ download/493150/data/qipscompetencies.pdf

17 Dumenco L, Monteiro K, George P, et al. An interactive quality improvement and patient safety workshop for first-year medical students. MedEdPORTAL 2018;14:10734.

18 Ryan Miller M, Tessa Winterton M, Hoffman WW. Building a whole new mind: an interprofessional experience in patient safety and quality improvement education using the IHI open school, 2014.

19 Reznek MA, Digiovine B, Kromrei H, et al. Quality education and safe systems training (QuESST): development and assessment of a comprehensive cross-disciplinary resident quality and patient safety curriculum. J Grad Med Educ 2010;2:222-7.

20 Gonzalo JD, Chuang $\mathrm{CH}$, Huang G, et al. The return of bedside rounds: an educational intervention. $J$ Gen Intern Med 2010;25:792-8

21 IHI patient safety online modules. Available: http://www.ihi.org/ education/IHIOpenSchool/Courses/Pages/default.aspx

22 Gonzalo JD, Heist BS, Duffy BL, et al. The value of bedside rounds: a multicenter qualitative study. Teach Learn Med 2013;25:326-33.

23 Gonzalo JD, Masters PA, Simons RJ, et al. Attending rounds and bedside case presentations: medical student and medicine resident experiences and attitudes. Teach Learn Med 2009;21:105-10.

24 Strauss A, Corbin JM. Basics of qualitative research. In: Grounded theory procedures and techniques. London: Sage Publications, Inc, 1990.

25 . Available: https://psnet.ahrq.gov/primer/improving-patient-safetyand-team-communication-through-daily-huddles

26 Lamba AR, Linn K, Fletcher KE. Identifying patient safety problems during team rounds: an ethnographic study. BMJ Qual Saf 2014;23:667-9.

27 Stickrath C, Noble M, Prochazka A, et al. Attending rounds in the current era: what is and is not happening. JAMA Intern Med 2013;173:1084-9.

28 Shoeb M, Khanna R, Fang M, et al. Internal medicine rounding practices and the accreditation Council for graduate medical education core competencies. J Hosp Med 2014;9:239-43.

29 Roh H, Park SJ, Kim T. Patient safety education to change medical students' attitudes and sense of responsibility. Med Teach 2015;37:908-14.

30 Tregunno D, Ginsburg L, Clarke B, et al. Integrating patient safety into health professionals' curricula: a qualitative study of medical, nursing and pharmacy faculty perspectives. BMJ Qual Saf 2014;23:257-64.

31 Ginsburg LR, Tregunno D, Norton PG. Self-Reported patient safety competence among new graduates in medicine, nursing and pharmacy. BMJ Qual Saf 2013;22:147-54.

32 Foundation LLlatNPS. Unmet needs: teaching physicians to provide safe patient care, 2010: 1-37. http://www npsf org/wp-content/ uploads/2011/10/LLI-Unmet-Needs-Report pdf 